\title{
Prevalence and factors associated with Group A rotavirus infection among children with acute diarrhea in Mwanza, Tanzania
}

\author{
Akwila Temu ${ }^{1}$, Erasmus Kamugisha ${ }^{2}$, Damas L Mwizamholya ${ }^{1}$, Aldofina Hokororo ${ }^{1}$, Jeremiah \\ Seni $^{3}$, Stephen E Mshana ${ }^{3}$ \\ ${ }^{1}$ Department of Pediatric and Child Health Bugando Medical Centre Box 1370 Mwanza, Tanzania \\ ${ }^{2}$ Department of Biochemistry Weill Bugando University College of Health Sciences BOX 1464 Mwanza, Tanzania \\ ${ }^{3}$ Department of Microbiology/ Immunology Weill Bugando University College of Health Sciences BOX 1464 \\ Mwanza, Tanzania
}

\begin{abstract}
Introduction: Rotavirus infections frequently cause acute gastroenteritis in humans and are the most important cause of severe dehydrating diarrhea in young children in both developed and developing countries.

Methodology: This was a prospective cross-sectional, hospital-based study on 300 children $\leq 5$ years with acute watery diarrhea who attended Bugando Medical Centre (BMC) and Nyamagana District hospital between May and November 2009. Stool specimens were tested for rotavirus infection using latex agglutination test. Data were cleaned and analyzed using SPSS 11.0.

Results: Of 300 children with acute watery diarrhea, 136 (45.3\%) were female and the mean age was 12.63 months (SD = 10.4). Sixty-two $(20.7 \%)$ children were found to have rotavirus infection. Of children with severe malnutrition three $(37.5 \%)$ were infected with rotavirus. Fifty-two (84\%) of children with rotavirus infection were below two years of age. Severe dehydration was present in $48(16 \%)$ children of whom $12(25 \%)$ were infected with rotavirus compared to $18(16.6 \%)$ of 109 children with no dehydration. Living next door to a child with diarrhea was highly associated with rotavirus infection $(43 \%$ versus $19 \%$; $=0.036)$. The mean hospital stay among children with rotavirus infection was 3.66 days versus 2.5 days for those without rotavirus $(\mathrm{p}=0.005)$.

Conclusion: Rotavirus infection is prevalent in Mwanza region and contributes to prolonged hospital stay. Proper education on hygiene to control diarrheal diseases among children should be emphasized. Extensive studies to determine the serotypes of rotavirus are warranted in the region before rotavirus vaccine is introduced.
\end{abstract}

Key words: Rotavirus; Mwanza; Tanzania

J Infect Dev Ctries 2012; 6(6):508-515.

(Received 27 December 2010 - Accepted 13 July 2011)

Copyright (C) 2012 Temu et al. This is an open-access article distributed under the Creative Commons Attribution License, which permits unrestricted use, distribution, and reproduction in any medium, provided the original work is properly cited

\section{Introduction}

Diarrheal diseases in children are a major public health concern in developing countries, including Tanzania. Diarrhea is a major cause of malnutrition in children, predisposing them to other infectious diseases that increase child mortality in these countries. Estimates of mortality in developing countries reveal 4.9 children per 1,000 per year die as a result of diarrheal illnesses in the first five years of life. This contributes to the childhood mortality in children younger than five years by $21 \%$, with most deaths occurring during the first year of life [1,2]. Acute gastroenteritis is among the most common illnesses of humankind and its associated morbidity and mortality are greatest among children and the elderly [3]. Rotaviruses are the most important cause of severe dehydrating diarrhoea in young children in both developed and developing countries [4]. Nearly all children are infected by a rotavirus at least once by the age five years [5]. Rotavirus is the most common cause of severe infantile diarrhea worldwide. According to an Institute of Medicine report, 130 million children are estimated to develop diarrhea due to rotavirus each year, resulting in more than 611,000 deaths [6].

Since rotavirus disease cannot be eliminated through improvements in water and sanitation, development of a safe and effective rotavirus vaccine has been a priority [7]. The prevalence of rotavirus infection, serotype characterization, and epidemiological distribution of rotaviral infections have been extensively studied in developed countries where rotavirus vaccination has been implemented. In developing countries, where rotavirus infection 
morbidity and mortality are high, data are lacking because no large comparable studies have been done.

In light of this problem, this study was done to understand the burden and epidemiology of rotavirus infection in Mwanza city, Tanzania. The results of this study will provide baseline information for future studies to determine serotypes of rotavirus in the community so that an appropriate vaccine can be introduced.

\section{Methodology}

This hospital-based, prospective cross-sectional study was conducted from May to December 2009. The study was performed at the Bugando Medical Center (BMC) and Nyamagana District Hospital, which are located in Mwanza City. BMC is one of the referral hospitals in Tanzania; located in the northwestern part of Tanzania, it serves as a referral, research and teaching hospital for six regions, namely Mwanza, Kagera, Shinyanga, Tabora, Mara, and Kigoma, with a de facto population of $13,000,000$. It has a bed capacity of 890 . The pediatric department has a bed capacity of 121 . The hospital has a clinical laboratory and a research laboratory. Nyamagana District Hospital serves Nyamagana district in Mwanza city; the hospital is about $5 \mathrm{~km}$ from the city centre and the pediatric department has 20 beds.

\section{Inclusion and exclusion criteria}

Children under five years of age with acute watery diarrhea whose parent/caregiver provided informed consent to participate and those admitted in the ward for less than 48 hours were recruited. Children with bloody diarrhea and acquisition of diarrheal disease during hospitalization were excluded.

\section{Sample size and sampling}

Sample size was calculated using the Kish and Lisle formula based on a prevalence of $24 \%$ [8]. A sample size of 300 children was obtained and recruited serially.

\section{Demographic and clinical information}

The following information for each patient was obtained using a standardized data collection form: place of residence, age, sex, presence and duration of fever and/or vomiting, consistency and frequency of stool, and duration of the current episode of diarrhea in past 24 hours. Other co-morbid conditions, feeding history, family social history, and history of a past illness with diarrhea were recorded. Participants were also asked if there was any history of a child's neighbor with diarrhea and this information was recorded. Clinical examination was done to elicit hydration status, fever (which was defined as an axillary temperature exceeding $37.5^{\circ} \mathrm{C}$ on admission) palmar pallor, wasting, bilateral pitting edema in lower limbs, lymph node enlargement, respiratory rate, and skin problems. Additional clinical information such as previous treatment given and laboratory investigations was obtained from inpatient records. Infants under two years of age were weighed using a $25 \mathrm{~kg}$ Salter hanging scales (CMS Weighing equipment, High Holborn, London, United Kingdom), while those above two years of age were weighed while standing on scales, with the scales adjusted to zero before each measurement. Children's weight was recorded to the nearest 100 grams. For infants less than 85 centimeters or those unable to stand alone, length was taken while lying on the measuring board; for walking children above 85 centimeters, height was measured while standing on the measuring board. The mid upper arm circumference was measured by a tape measure in the left arm among children aged 6 to59 months; the reading was recorded to the nearest $0.1 \mathrm{~cm}$.

\section{Specimen collection and laboratory procedures}

Stool specimens were collected on the same day of enrollment using a wide-mouth screw cap sterile container (HIMedia laboratories Pvt. Limited, Mumbai, India). Specimens were sent to the laboratory for analysis on the same day; most of the specimens were analyzed within 2 hours of collection. Fecal samples were processed as described in the instructions provided with the Rotalex kit (Orion Diagnostic, Finland) manual. In short, stool samples were diluted in a ratio of $1: 10$ with Rotalex buffer and mixed using vortex mixer and the suspension was allowed to stand for about 30 minutes at room temperature, followed by centrifugation for 30 minutes at $3000 \mathrm{rpm}$. Two separate drops of $50 \mu 1$ each from the above supernatant were applied on the slide. Rotalex reagent was added to one drop, and Rotalex control latex reagent was added to the other drop. The slide was gently shaken back and forth for 2 minutes. Development of agglutination in Rotalex reagent was treated as positive (Rotalex manual). Positive and negative controls were run with each test. 


\section{Data management}

Data were coded and entered into the logbooks and then into the computer and analyzed using the Statistical Package for the Social Sciences (SPSS for Windows version 11.0, IBM, Chicago, Ill, USA). Comparison of proportions and statistical significance were tested by using the Chi-square test. A $p$ value less than 0.05 was considered statistically significant

\section{Ethical considerations}

The study proposal was submitted at the Bugando Medical Centre/Weill Bugando University College of Health Sciences (BMC/WBUCHS) ethics committee for ethical clearance. Parents/caretakers were requested to sign a consent form for their willingness to participate in the study.

\section{Study limitations}

No typing was done to determine the serotype and genotype of rotavirus. Recall bias could also affect the results.

\section{Results}

General patient characteristics

A total of 300 children with acute watery diarrhea were enrolled in the study; males formed the majority of the study population $164(54.6 \%)$. From BMC $139(46.3 \%)$ patients were enrolled while 161 $(53.7 \%)$ patients were enrolled from Nyamagana District Hospital. Sixty-two $(20.7 \%)$ of children were found to be infected with rotavirus; of the 62 children with rotavirus infection $52(84 \%)$ were below two years of age (Table 1).

\section{Nutritional status}

In the weight for height classification, most children $(\mathrm{n}=271)$ had a normal nutritional status, 54 $(20 \%)$ of whom had rotavirus infection. The proportion of rotavirus positive children increased with increasing severity of malnutrition $(24 \%$ and $37.5 \%$ for moderate malnutrition and severe malnutrition respectively). However, this trend was not statistically significant $(\mathrm{p}$ value $=0.449)$ (Table 2)

\section{HIV status and rotavirus infection}

All 300 enrolled patients were questioned about HIV serostatus; however, HIV testing was not performed. The responses indicated that the majority of the patients were negative $(\mathrm{n}=212), 9$ were positive, and 79 were unknown. Rotavirus infection was found in $43(20 \%)$ and in $2(22.2 \%)$ among HIV negative and positive patients respectively. The difference in these proportions was not statistically significant, $\mathrm{p}$ value $=0.991$ (Table 2$)$.

\section{Clinical characteristics}

All enrolled patients had diarrhea on admission to the study. In addition, 157 patients presented with vomiting, 208 with fever, 37 with cough, 12 with oral sores, 48 with severe dehydration, 143 with some dehydration and 109 with no dehydration. Considering risks for diarrhea (Table 3), 71 patients had a past episode of diarrhea, out of whom 17 $(23.9 \%)$ had rotavirus infection, and history of child's neighbour with diarrhea was noted in 14 patients, out of whom $6(43 \%)$ had rotavirus infection $(\mathrm{p}$ value $=$ 0.036).

\section{Treatment and outcome}

Dehydration was uniformly distributed between patients with rotavirus infection and those without the infection $(\mathrm{p}=0.404)$. All patients (48) with severe dehydration were given intravenous fluid (IVF) whether they tested positive for rotavirus or not, and all patients with some dehydration and no dehydration were treated with oral rehydration therapy (ORT). Out of 300 children, 296 (98.3\%) including all $62(20.6 \%)$ patients with rotavirus infection improved after treatment. Four patients died and one patient was referred from Nyamagana district hospital. Children with rotavirus infection had prolonged hospital stay (mean 3.66 days compared to 2.5 days for those with no rotavirus infection; $\mathrm{p}=$ $0.005)$.

\section{Discussion}

\section{Prevalence of rotavirus infection}

Acute watery diarrhea can be linked to various pathogens among which viruses are responsible for more than half of the cases [9]. Rotavirus accounts for more than $50 \%$ of severe diseases. Rotavirus group A, which accounts for more than $90 \%$ of rotavirus gastroenteritis in humans, is endemic worldwide [10]. Our study found that of 300 enrolled diarrhea patients, $62(20.7 \%)$ tested positive for rotavirus. These findings are similar to those of other published studies in Tanzania [11]. In a more recent study in three hospitals in Dar es Salaam by Moyo et al. 2007 using EIA test on 270 stool samples, the prevalence of rotavirus infection was $18 \%$ [12]. The difference between the studies can possibly be due to their respective durations, as the Dar es Salaam study 
Table 1. Age groups and rotavirus infection

\begin{tabular}{lcc}
\hline Age group (n) & N (\%) & Rotavirus +VE n (\%) \\
\hline$<6$ months & 60 & $7(11.7 \%)$ \\
6 to $<12$ months & 124 & $25(20.2 \%)$ \\
12 to $<24$ months & 72 & $20(27.8 \%)$ \\
24 to $<36$ months & 26 & $3(11.5 \%)$ \\
36 to $<48$ months & 10 & $5(50 \%)$ \\
48 to $<60$ months & 8 & $2(25 \%)$ \\
Total & $\mathbf{3 0 0}$ & $\mathbf{6 2}(\mathbf{2 0 . 7 \% )}$ \\
\hline
\end{tabular}

Table 2. Characteristics of study population

\begin{tabular}{|c|c|c|c|c|c|}
\hline Parameter & $\mathrm{N}$ & $\%$ Rotavirus + & \%Rotavirus - & $\mathrm{P}=$ value & $95 \% \mathrm{C} 1$ \\
\hline \multicolumn{6}{|l|}{ Age (Months) } \\
\hline Mean & 12.63 & NA & NA & NA & NA \\
\hline SD & 10.374 & NA & NA & NA & NA \\
\hline \multicolumn{6}{|l|}{$S E X$} \\
\hline Female & 136 & $31(23 \%)$ & $105(77 \%)$ & 0.407 & $16.3-30.4$ \\
\hline Male & 164 & $31(19 \%)$ & $133(81 \%)$ & & $13.4-25.4$ \\
\hline \multicolumn{6}{|l|}{ Nutrition Status } \\
\hline Normal & 271 & $54(20 \%)$ & $217(80 \%)$ & 0.449 & $15.5-25.0$ \\
\hline Moderate & 21 & $5(24 \%)$ & $16(76 \%)$ & & $9.3-45.1$ \\
\hline Severe & 8 & $3(37.5 \%)$ & $5(62.5 \%)$ & & $10.6-72.2$ \\
\hline \multicolumn{6}{|l|}{ HIV status } \\
\hline Negative & 212 & $43(20 \%)$ & $160(80 \%)$ & 0.991 & $15.3-26.1$ \\
\hline Positive & 9 & $2(22.2 \%)$ & $7(77.8 \%)$ & & $3.9-56.2$ \\
\hline Not known & 79 & $17(21.5 \%)$ & $62(78.5 \%)$ & & $13.5-31.6$ \\
\hline \multicolumn{6}{|l|}{ Occupation } \\
\hline Business & 46 & $8(17.4 \%)$ & $38(82.6 \%)$ & 0.871 & $8.4-30.4$ \\
\hline Employed & 22 & $3(13.6 \%)$ & $19(86.4 \%)$ & & $3.6-32.8$ \\
\hline Peasant & 47 & $10(21.3 \%)$ & 37 (78.7\%) & & $11.3-34.7$ \\
\hline Self-employed & 28 & $6(21.4 \%)$ & $22(78.6 \%)$ & & $9.2-39.3$ \\
\hline Housewife & 157 & $35(22.3 \%)$ & $122(77.7 \%)$ & & $16.3-29.3$ \\
\hline
\end{tabular}


Table 3. History of children + rotavirus infections

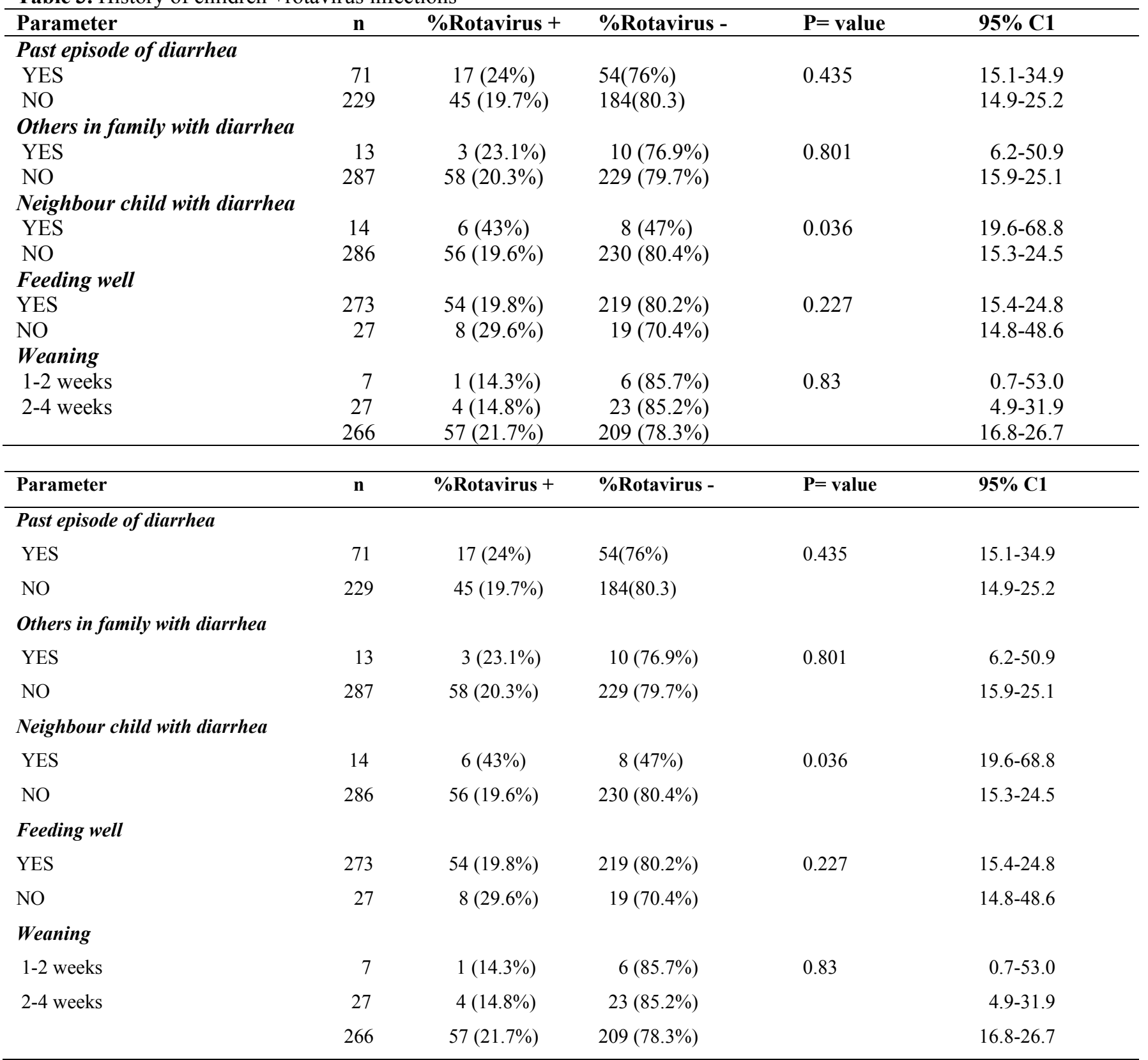

was done for three months, while our study spanned for seven months, and one cannot estimate an annual prevalence based on short studies. This difference in prevalence in the same country has also been reported in another study [13]. The rotavirus prevalence in our study is similar to those found in other Africa Network countries, where prevalence ranged from $19 \%$ in Senegal (the lowest), to $21 \%$ in Cote d'Ivoire, to of $52 \%$ (the highest) in Ghana [8]. Our study prevalence was also comparable to that of an investigation in Vietnam where the reported prevalence was $21 \%$ [14], and also similar to the prevalence observed in other studies in developed countries such as Spain (17.1\%) [15] and Israel $(18.4 \%)$ [16].

Demographic characteristics of children with rotavirus infection

In our study, males dominated in the total number of cases as compared to females (Table 2). There was no sex predilection when it came to rotavirus positivity for males. Similar findings were 
reported in a study in Chennai India, where Saravanan et al., found no predilection for the male sex, which was in contrast to the observations in many other cited studies, wherein a preponderance of infection was observed in male children ranging from $60 \%$ to $77 \%$ [17]. The male predilection could be explained by genetic and immunological factors in that, although both sexes are infected at the same rate, males are more prone to develop a severe form of diarrhea necessitating hospital admission [7].

The age of patients ranged from 0 months to 59 months with a mean age of 12.63 months (standard deviation 10.374). Most patients $(\mathrm{n}=184,61.3 \%)$ were aged 12 months or less. This is an important finding as it shows that most infections in our locality occur in infants, where morbidity and mortality from diarrhea is high [8]. The finding is also important in the timing for rotavirus vaccines in our country once started. The finding is consistent with those in all tropical countries where it is reported that diarrhea and rotavirus diarrhea tend to occur early in life, and symptomatic infection rates are highest in children under two years of age and decrease progressively towards four to five years $[18,19]$.

The highest incidence of rotavirus diarrhea in this study occurred in children aged 6 to 12 months. This is similar to the incidence in other developing countries where rotavirus is a significant pathogen among infants aged less than 12 months [20]. Within the group of those infants below one year, there is a significant variation in the number of diarrhea and rotavirus infected cases from the neonatal period to the early and late infancy group. Infection in newborns, although common, is often associated with mild or asymptomatic disease [21]. It was found that in the first three months of life there were few cases of diarrhea. This variation is explained by the feeding and development of the infants [17]. During the neonatal period, most infants are protected by passively acquired maternal antibodies. Exclusive breastfeeding decreases the chance of contamination with rotavirus. The inability of neonates to infect themselves through their fingers may partly explain the lower rate of both diarrhea and rotavirus at this age group. Neonates on exclusive breastfeeding pass several soft stools per day, so even if they are infected at this young age, the infection can be asymptomatic or can be mild, hence undetected. The sharp increase in the incidence of diarrhea and rotavirus in the subsequent age groups is explained partly by the waning passive immunity [17] and that the early exposure to rotavirus infection during the neonatal life does not protect against future episodes as immunity is not acquired at this early age. Furthermore, the introduction of complementary and weaning feeds increase contamination of the gut by pathogens. During the same period the body starts to acquire immunity. At the age of one year, there is sound active immunity to protect against most diarrheal pathogens. Children 12 months to 5 years can still get diarrhea from rotavirus and other pathogens, but the episodes may be asymptomatic or will tend to be less severe, so patients may end up staying at home or visiting the hospital as outpatients. These findings have been reported by studies elsewhere, especially in tropical countries where rotavirus and other diarrheal pathogens are acquired early in life $[12,13]$. It was reported further that the rate of rotavirus infection increased with age in the first year of life. There was a study in Nigeria which showed that the younger the age the higher the risk for rotavirus induced diarrhea, with the highest burden detected in children less than six months [13]. In another study in Turkey rotavirus infection affected mainly children 6 to 23 months [22]. These findings are important for determining timing of vaccination against rotavirus in different parts of the world [23].

\section{Nutritional status of children with rotavirus diarrhoea}

Using the weight/height and the MUAC classifications, there was no statistically significant difference in rotavirus infection proportions among children with normal and those with abnormal nutritional status. This finding conforms with the basic pathogenesis of rotavirus diarrhea, i.e., that it can affect children under the age of five years regardless of their nutritional status, and protection is determined by acquired immunity from prior exposure to rotavirus infection or vaccination against rotavirus [24, 25].

The duration of hospital stay was slightly longer among malnourished than well-nourished children. Though the differences are not statistically significant, rotavirus diarrhea morbidity was higher in the malnourished children than among wellnourished children. Malnourished children have defective humoral and cellular immunity; hence they are more liable to suffer from prolonged diarrhoea, and may become chronically infected by rotavirus [24]. 


\section{Treatment outcome}

The mean hospital stay among children with rotavirus infection and those without rotavirus infection was 3.7 days and 2.5 days respectively. It was found that patients with rotavirus infection stayed much longer than patients without rotavirus infection. Duration of hospitalization due to rotavirus infection usually ranges from 2 to 14 days with a mean of 4 days. This finding is similar to the pathogenesis of rotavirus diarrhoea where it has been documented that an episode usually lasts between 4 to 9 days [23].

Our study corroborated the findings of another study [19] which showed that rotavirus is a disease with very high morbidity and high socio-economic implications in terms of medical costs and time spent in hospital. If well managed, its mortality can be minimal and rarely cause complications. In the study all patients with rotavirus infection were successfully treated using the Integrated Management of Child Illness (IMCI) guidelines and recovered. However, the risk of death is still high in developing countries as compared to Europe and the United States [19, 26, 27].

\section{Conclusion}

Rotavirus infection is a major cause of severe dehydrating diarrhea in young children. Prevalent in Mwanza city and in other parts of Tanzania and other developing countries, it has high morbidity and may contribute to prolonged hospital stays for infected children. The presence of diarrhea in a neighboring child increases risk of rotavirus infection. Rotavirus infection is not associated with nutritional status. Prognosis of rotavirus diarrhoea is good if patients are well managed. Extensive studies to determine the serotypes of rotavirus are warranted in the region before the introduction of rotavirus vaccine. Proper education on hygiene to control diarrheal diseases among children should be emphasized.

\section{Acknowledgement}

The authors would like to acknowledge the technical support provided by the members of the Departments of

Microbiology/Immunology of WBUCHS and Institute of Medical Microbiology Giessen. We thank Mary Louise Shushu, Hezron Bassu and Ms Elizabeth John, for their excellent technical assistance. This work was supported by a research grant from WBUCHS to SEM.

\section{References}

1. Parashar UD, Glass R, Ivanoff B (1999) Current status and future priorities for rotavirus vaccine development; evaluation, and implementation in developing countries. Vaccine 17: 2207-2222.

2. Bern C, Martines J, de Joysa I, Glass R.I (1992) The magnitude of the global problem of diarhoea disease. Bull World health Organ 70: 705-714.

3. Wilhelmi I, Roman E, Sánchez-Fauquier A (2003) Viruses causing gastroenteritis. Clin Microbiol Infect 9: 247-262.

4. Glass RI, Parashar UD, Bresee JS, Turcios R, Fischer TK, Widdowson MA (2006) Rotavirus vaccines: current prospects and future challenges. Lancet 368: 323-332.

5. Forster J, Hammerschmidt $T$ (2007) Burden of acute rotavirus gastroenteritis (RV-AGE) in Germany: a comparison of federal statistics and epidemiological data. Gesundheitswese 69: 227-232.

6. Institute of Medicine (1986) The prospect of immunization against rotavirus. In $\mathrm{New}$ vaccines for development: diseases of importance in developing countries, 2nd ed. National Academy Press, Washington, D.C. p. D-13-1-D13-2.

7. Fischer TK, Viboud C, Parashar U (2007) Hospitalizations and deaths from diarrhea and rotavirus among children < 5 years of age in the United States, 1993-2003. J Infect Dis 195: 1117-1125.

8. Cunliffe NA, Kilgore PE, Bresee JS, Steele AD, Luo N, Hart CA, RI Glass (1998) Epidemiology of rotavirus diarrhoea in Africa: a review to assess the need for rotavirus immunization. Bull World Health Organ 76: 525-537.

9. Alain S and Denis F (2007) Epidemiology of infectious acute diarrhoea in France and Europe. Arch Pediatr 14 Suppl 3: S132-44.

10. Bányai K, Judit D, James G, Miren I, Julianna K, József K (2007) EuroRota_Net--European rotavirus strain surveillance network established with Hungarian participation. György Szucs Orv Hetil 28: 2043-2045

11. Mhalu FS, Myrmel H, Msengi A, Haukenes G (1998) Prevalence of infection with rotavirus and enteric adenoviruses among children in Tanzania. NIPH Ann 11: 37.

12. Moyo S J, Njolstad G, Kirsti V, Matee M I, Kitundu J, Maselle SY (2007) Prevalence of enteropathogenic viruses and molecular characterization of group A rotavirus among children with diarrhea in Dar es Salaam Tanzania. BMC Public Health 7: 359.

13. Adah M I, Abel W, Koki T (2001) Molecular Epidemiology of Rotaviruses in Nigeria: Detection of Unusual Strains with G2P 6 and G8P 1 Specificities. J Clinic Microbiol 39: 39693975.

14. Hoshino Y, Kapikian AZ (1996), Classification of rotavirus VP4 and VP7 serotypes. Arch.Virol Suppl 12: 99-111.

15. López-de-Andrés A, Jiménez-García R, Carrasco-Garrido P, Alvaro-Meca A, Galarza PG, de Miguel AG (2008) Hospitalizations associated with rotavirus gastroenteritis in Spain, 2001-2005. BMC Public Health 8: 109.

16. Grisaru-Soen G, Engelhard D, Pearl S, Schlesinger Y, Shtein M, Ashkenazi S (2008) Hospitalizations associated with rotavirus gastroenteritis in Israel a retrospective study. Harefuah. 147: 8-11.

17. Saranavan P, Ananthan S, Ananthasubramanian M (2004) Rotavirus infection among infants and young children in Chennai, South India. Indian journal of Medical Microbiology. 22: 212-221.

18. Koopmans M and Brown D (1999) Seasonality and diversity of Group A. rotaviruses in Europe. Acta paediatrica 88 Suppl 426: 14-19. 
19. Guerrant RL, Kosec M, Moore S, Lorntz B, Brantley R, Lima AA (2002) Magnitude and Impact of diarrheal disease. Arch Med Res 33: 351-355.

20. Chouikha A, Fodha I, Peenze M, De Beer J, Dewar A, Messadi F, MB Taylor, A Trabelsi and D Steele (2008) Identification of viral agents causing diarrhoea among children in the Eastern centre of Tunisia. J. Med. Virol 78: 1198-1203.

21. Steele D, Reynecke E, de Beer M, Bos P, Smuts I (2002) Characterization of rotavirus infection in a hospital neonatal unit in Pretoria. South Africa. J Trop Pediatr 48: 167-171.

22. Bozdayi G, Dogan B, Dalgic B, Bostanci I, Sari S, Battaloglu NO, Rota S, Dallar Y, Nishizono A, Nakagomi O, Ahmed K (2008) Diversity of human rotavirus G9 among children in Turkey. J Med Virol 80733-40.

23. Bresee JS, Hummelman E, Nelson EA, Glass RI (2005). Rotavirus in Asia: The value of surveillance for informing decisions about the introduction of new vaccines. J Infect Dis 192 Suppl 1: S1.

24. Hussein A and Hassan M (2000) Rotavirus infection among hospitalized children with acute watery diarrhoea in BasrahIraq. Bahrain Medical Bulletin 22: 170-173.
25. Kiulia N, Julia K, Ina Peenze, Atunga N, Rachael N, Steele $\mathrm{D}$, and Jason M. Rotavirus infection among HIV-infected children in Nairobi, Kenya. J Tropical Pediatrics 55: 318323.

26. Cunliffe NA, Gondwe JS, Graham SM, Thindwa BD, Dove W, Broadhead RL, et al (2001) Rotavirus strain diversity in Blantyre, Malawi, from 1997 to 1999. J Clin Microbiol 39: 836-843.

27. Diggle L (2007) Rotavirus diarrhoea and future prospects for prevention. Br. J. Nurs 16: 970-974.

\section{Corresponding author}

Stephen E Mshana

Department of Microbiology/Immunology

BOX 1464

Mwanza, Tanzania

Telephone: +255 28-250-0881

Email:mshana72@yahoo.com

Conflict of interests: No conflict of interests is declared. 